\title{
RIESZ SEQUENCES AND ARITHMETIC PROGRESSIONS
}

\author{
ITAY LONDNER AND ALEXANDER OLEVSKII
}

\begin{abstract}
Given a set $\mathcal{S}$ of positive measure on the circle and a set of integers $\Lambda$, one may consider the family of exponentials $E(\Lambda):=\left\{e^{i \lambda t}\right\}_{\lambda \in \Lambda}$ and ask whether it is a Riesz sequence in the space $L^{2}(\mathcal{S})$.

We focus on this question in connection with some arithmetic properties of the set of frequencies.

Improving a result of Bownik and Speegle ([3], Thm. 4.16), we construct a set $\mathcal{S}$ such that $E(\Lambda)$ is never a Riesz sequence if $\Lambda$ contains arbitrarily long arithmetic progressions of length $N$ and step $\ell=O\left(N^{1-\varepsilon}\right)$. On the other hand, we prove that every set $\mathcal{S}$ admits a Riesz sequence $E(\Lambda)$ such that $\Lambda$ does contain arbitrarily long arithmetic progressions of length $N$ and step $\ell=O(N)$.
\end{abstract}

\section{INTRODUCTION}

We use below the following notation:

$\Lambda$ - A set of integers.

$\mathcal{S}$ - A set of positive measure on the circle $\mathbb{T}$.

$|\mathcal{S}|$ - The Lebesgue measure of $\mathcal{S}$.

For $A, B \subset \mathbb{R}, x \in \mathbb{R}$ we let

$$
A+B:=\{\alpha+\beta \mid \alpha \in A, \beta \in B\}, x \cdot A:=\{x \cdot \alpha \mid \alpha \in A\} .
$$

The research is supported in part by the Israel Science Foundation

2010 Mathematics Subject Classification. Primary 42C15; Secondary 42A38.

Key words and phrases. Riesz sequences, Arithmetic progressions. 
A sequence of elements in a Hilbert space $\left\{\varphi_{i}\right\}_{i \in I} \subset \mathcal{H}$ is called a Riesz sequence $(\mathrm{RS})$ if there are positive constants $c, C$ s.t. the inequalities

$$
c \sum_{i \in I}\left|a_{i}\right|^{2} \leq\left\|\sum_{i \in I} a_{i} \varphi_{i}\right\|^{2} \leq C \sum_{i \in I}\left|a_{i}\right|^{2},
$$

hold for every finite sequence of scalars $\left\{a_{i}\right\}_{i \in I}$.

Given $\Lambda \subset \mathbb{Z}$ we denote

$$
E(\Lambda):=\left\{e^{i \lambda t}\right\}_{\lambda \in \Lambda}
$$

The following result is classical (see [9], p.203, Lemma 6.5):

If $\Lambda=\left\{\lambda_{n}\right\}_{n \in \mathbb{N}} \subset \mathbb{Z}$ is lacunary in the sense of Hadamard, i.e. satisfies

$$
\frac{\lambda_{n+1}}{\lambda_{n}} \geq q>1, n \in \mathbb{N}
$$

then $E(\Lambda)$ forms a $R S$ in $L^{2}(S)$, for every $\mathcal{S} \subset \mathbb{T}$ with $|\mathcal{S}|>0$.

The following generalization is due to I.M. Miheev ([7], Thm. 7):

If $E(\Lambda)$ is an $S_{p}-$ system for some $p>2$, i.e. satisfies

$$
\left\|\sum_{\lambda \in \Lambda} a_{\lambda} e^{i \lambda t}\right\|_{L^{p}(\mathbb{T})} \leq C\left\|\sum_{\lambda \in \Lambda} a_{\lambda} e^{i \lambda t}\right\|_{L^{2}(\mathbb{T})},
$$

with some $C>0$, for every finite sequence of scalars $\left\{a_{\lambda}\right\}_{\lambda \in \Lambda}$, then it forms a $R S$ in $L^{2}(\mathcal{S})$, for every $\mathcal{S} \subset \mathbb{T}$ with $|\mathcal{S}|>0$.

J. Bourgain and L. Tzafriri proved the following result as a consequence of their "restricted invertibility theorem" ([2], Thm. 2.2):

Given $\mathcal{S} \subset \mathbb{T}$, there is a $R S E(\Lambda)$ s.t. $\Lambda$ is a set of integers with positive asymptotic density

$$
\operatorname{dens} \Lambda:=\lim _{N \rightarrow \infty} \frac{\#\{\Lambda \cap[-N, N]\}}{2 N}>C|\mathcal{S}|
$$


(Here and below $C$ denotes positive absolute constants, which might be different from one another).

W. Lawton ([5], Cor. 2.1), assuming the Feichtinger conjecture for exponentials, proved the following proposition:

$(L)$ For every $\mathcal{S}$ there is a $R S E(\Lambda)$ s.t. the set of frequencies $\Lambda \subset \mathbb{Z}$ is syndetic, that is $\Lambda+\{0, \ldots, n-1\}=\mathbb{Z}$ for some $n \in \mathbb{N}$.

Recall that the Feichtinger conjecture says that every bounded frame in a Hilbert space can be decomposed in a finite family of $\mathrm{RS}$. This claim turned out to be equivalent to the Kadison-Singer conjecture (see [4]). The last conjecture has been proved recently by A. Marcus, D. Spielman and N. Srivastava (see [6]), so proposition $(L)$ holds unconditionally.

Notice that in some results above the system $E(\Lambda)$ serves as RS for all sets $\mathcal{S}$; however the set of frequencies $\Lambda$ is quite sparse there. In others $\Lambda$ is rather dense but it works for $\mathcal{S}$ given in advance.

It was asked in [8] whether one can somehow combine the density and "universality" properties. It turned out this is indeed possible. A set $\Lambda \subset \mathbb{R}$ has been constructed in that paper such that the exponential system $E(\Lambda)$ forms a RS in $L^{2}(\mathcal{S})$ for any open set $\mathcal{S}$ of a given measure, and the set of frequencies has optimal density, proportional to $|\mathcal{S}|$. This is not true for nowhere dense sets (see [8]).

\section{Results}

In this paper we consider sets of frequencies $\Lambda$ which contain arbitrarily long arithmetic progressions. Below we denote the length of a progression by $N$, by $\ell$ we denote its step. Given $\Lambda$ which contains arbitrarily long arithmetic progressions there exists a set $\mathcal{S} \subset \mathbb{T}$ of positive measure so that $E(\Lambda)$ is not a $\mathrm{RS}$ in $L^{2}(\mathcal{S})$ (see [7]). 
In the case $\ell$ grows slowly with respect to $N$, one can define $\mathcal{S}$ independent of $\Lambda$. A quantitative version of such a result was proved in [3]:

There exists a set $\mathcal{S}$ such that $E(\Lambda)$ is not a $R S$ in $L^{2}(\mathcal{S})$ whenever $\Lambda$ contains arithmetic progressions of length $N_{j}\left(N_{1}<N_{2}<\ldots\right)$ and the corresponding step $\ell_{j}=o\left(N_{j}^{1 / 2} \log ^{-3} N_{j}\right)$.

The proof is based on some estimates of the discrepancy of sequences of the form $\{\alpha k\}_{k \in \mathbb{N}}$ on the circle.

Using a different approach we prove a stronger result:

Theorem 1. There exists a set $\mathcal{S} \subset \mathbb{T}$ such that if a set $\Lambda \subset \mathbb{Z}$ contains arithmetic progressions of length $N\left(=N_{1}<N_{2}<N_{3}<\ldots\right)$ with corresponding step $\ell=$ $O\left(N^{\alpha}\right), \alpha<1$, then $E(\Lambda)$ is not a $R S$ in $L^{2}(\mathcal{S})$.

Here one can construct $\mathcal{S}$ not depending on $\alpha$ and with arbitrarily small measure of the complement.

The next theorem shows that the result is sharp.

Theorem 2. Given a set $\mathcal{S} \subset \mathbb{T}$ of positive measure, there is a set of frequencies $\Lambda \subset \mathbb{Z}$ so that:

(i) For infinitely many $N$ 's $\Lambda$ contains an arithmetic progression of length $N$ and step $\ell=O(N)$.

(ii) The system of exponentials $E(\Lambda)$ forms a $R S$ in $L^{2}(\mathcal{S})$.

Increasing slightly the bound for $\ell$, one can get a version of Theorem 2 which admits a progression of any length:

Theorem 3. Given $\mathcal{S}$ one can find $\Lambda$ with the property (ii) above and s.t.

(i') For every $\alpha>1$ and for every $N \in \mathbb{N}$ it contains an arithmetic progression of length $N$ and step $\ell<C(\alpha) N^{\alpha}$. 


\section{Proof of Theorem 1}

Proof. Fix $\varepsilon>0$. Take a decreasing sequence of positive numbers $\{\delta(\ell)\}_{\ell \in \mathbb{N}}$ s.t.

$$
\begin{aligned}
& \text { (a) } \sum_{\ell \in \mathbb{N}} \delta(\ell)<\frac{\varepsilon}{2} \\
& \text { (b) } \delta(\ell) \cdot \ell^{1 / \alpha} \underset{\ell \rightarrow \infty}{\longrightarrow} \infty \quad \forall \alpha \in(0,1)
\end{aligned}
$$

For every $\ell \in \mathbb{N}$ set $I_{\ell}=(-\delta(\ell), \delta(\ell))$ and let $\tilde{I}_{\ell}$ be the $2 \pi$-periodic extension of $I_{\ell}$, i.e.

$$
\tilde{I}_{\ell}=\bigcup_{k \in \mathbb{Z}}\left(I_{\ell}+2 \pi k\right)
$$

We define

$$
I_{[\ell]}=\left(\frac{1}{\ell} \cdot \tilde{I}_{\ell}\right) \cap[-\pi, \pi] \text { and } \mathcal{S}=\mathbb{T} \backslash \bigcup_{\ell \in \mathbb{N}} I_{[\ell]}=\left(\bigcup_{\ell \in \mathbb{N}} I_{[\ell]}\right)^{c},
$$

whence we get that

$$
|\mathcal{S}| \geq 1-\sum_{\ell=1}^{\infty}\left|I_{[\ell]}\right|=1-\sum_{\ell=1}^{\infty} 2 \delta(\ell)>1-\varepsilon .
$$

Fix $\alpha<1$ and let $\Lambda \subset \mathbb{Z}$ be such that one can find arbitrarily large $N \in \mathbb{N}$ for which

$$
\{M+\ell, \ldots, M+N \cdot \ell\} \subset \Lambda,
$$

with some $M=M(N) \in \mathbb{Z}, \ell=\ell(N) \in \mathbb{N}$ and

$$
\ell<C(\alpha) N^{\alpha}
$$


Recall that by (1) we have $t \in I_{[\ell]}$ if and only if $t \ell \in \tilde{I}_{\ell} \cap[-\pi \ell, \pi \ell]$. Since $\mathcal{S}$ lies inside the complement of $I_{[\ell]}$ we get

$$
\begin{gathered}
\int_{\mathcal{S}}\left|\sum_{k=1}^{N} c(k) e^{i(M+k \ell) t}\right|^{2} \frac{d t}{2 \pi} \leq \int_{I_{[\ell]}^{c}}\left|\sum_{k=1}^{N} c(k) e^{i(M+k \ell) t}\right|^{2} \frac{d t}{2 \pi}= \\
=\int_{[-\pi \ell, \pi \ell] \tilde{I}_{\ell}}\left|\sum_{k=1}^{N} c(k) e^{i k \tau}\right|^{2} \frac{d \tau}{2 \pi \ell}=\int_{I_{\ell}^{c}}\left|\sum_{k=1}^{N} c(k) e^{i k \tau}\right|^{2} \frac{d \tau}{2 \pi} .
\end{gathered}
$$

Therefore, in order to complete the proof, it is enough to show that $\left\|\sum_{k=1}^{N} c(k) e^{i k \tau}\right\| \|_{L^{2}\left(I_{\ell}^{c}\right)}$ can be made arbitrarily small while keeping $\sum_{k=1}^{N}|c(k)|^{2}$ bounded away from zero. This observation allows us to reformulate the problem as a norm concentration problem of trigonometric polynomials of degree $N$ on the interval $I_{\ell}$.

Let $P_{N}(t)=\frac{1}{\sqrt{N}} \sum_{k=1}^{N} e^{i k t}$, so $\left\|P_{N}\right\|_{L^{2}(\mathbb{T})}=1$. Moreover, for every $t \in \mathbb{T}$ we have $\left|P_{N}(t)\right| \leq \frac{1}{\sqrt{N} \sin \frac{t}{2}}$, hence

$$
\int_{I_{\ell}^{c}}\left|P_{N}(t)\right|^{2} \frac{d t}{2 \pi} \leq \frac{1}{N} \int_{\delta(\ell)}^{\pi} \frac{d t}{\sin ^{2} \frac{t}{2}}<\frac{C}{N} \int_{\delta(\ell)}^{\pi} \frac{d t}{t^{2}}<\frac{C}{\delta(\ell) N}<\frac{C(\alpha)}{\delta(\ell) \ell^{1 / \alpha}}
$$

where last inequality holds for every $N$ for which (2) holds. Using condition (b) we see that indeed last term can be made arbitrarily small, and so $E(\Lambda)$ is not a $\mathrm{RS}$ in $L^{2}(\mathcal{S})$.

\section{Proof of THEOREM 2}

For $n \in \mathbb{N}$ we define

$$
B_{n}:=\left\{n, 2 n, \ldots, n^{2}\right\}
$$


Lemma 4. Let $\mathcal{P}$ be the set of all prime numbers. Then the blocks $\left\{B_{p}\right\}_{p \in \mathcal{P}}$ are pairwise disjoint.

Proof. Let $p<q$ be prime numbers. Notice that a number $a \in B_{p} \cap B_{q}$ if and only if there exist $1 \leq m \leq p$ and $1 \leq k \leq q$ s.t.

$$
a=m p=k q
$$

which is possible only if $q$ divides $m$. But since $m<q$ this cannot happen and so such $a$ does not exist.

Lemma 5. Let $\{a(n)\}_{n \in \mathbb{N}}$ a sequence of non-negative numbers s.t. $\sum_{n=1}^{\infty} a(n) \leq 1$. Then for every $\varepsilon>0$ there exist infinitely many $n \in \mathbb{N}$ s.t.

$$
\sum_{\ell=1}^{n} a(\ell n)<\frac{\varepsilon}{n} .
$$

Proof. By Lemma 4 we may write

$$
\sum_{n=1}^{\infty} a(n) \geq \sum_{p \in \mathcal{P}} \sum_{\ell=1}^{p} a(\ell p) .
$$

Assuming the contrary for some $\varepsilon$, i.e. for all but finitely many $p \in \mathcal{P}$ we have $\sum_{\ell=1}^{p} a(\ell p) \geq \frac{\varepsilon}{p}$, we get a contradiction to the well-known fact that $\sum_{p \in \mathcal{P}} \frac{1}{p}=\infty$.

Corollary 6. For every $\varepsilon>0$ there exist infinitely many $n \in \mathbb{N}$ s.t.

$$
\sum_{\substack{\lambda, \mu \in B_{n} \\ \mu<\lambda}} a(\lambda-\mu)<\varepsilon .
$$

Proof. Every $\mu<\lambda$ from $B_{n}$ must take the form

$$
\begin{aligned}
& \lambda=k n \\
& \mu=k^{\prime} n
\end{aligned}, 1 \leq k^{\prime}<k \leq n,
$$


hence $\lambda-\mu=\ell n, \ell \in\{1,2, \ldots n-1\}$. From Lemma 5 we get for infinitely many $n \in \mathbb{N}$

$$
\sum_{\substack{\lambda, \mu \in B_{n} \\ \mu<\lambda}} a(\lambda-\mu)=\sum_{\ell=1}^{n}(n-\ell) a(\ell n) \leq n \sum_{\ell=1}^{n} a(\ell n)<\varepsilon
$$

Given $B \subset \mathbb{R}$, we say that a positive number $\gamma$ is a lower Riesz bound (in $L^{2}(\mathcal{S})$ ) for the sequence $E(B)$ if the inequality

$$
\left\|\sum_{\lambda \in B} c(\lambda) e^{i \lambda t}\right\|_{L^{2}(\mathcal{S})}^{2} \geq \gamma \sum_{\lambda \in B}|c(\lambda)|^{2},
$$

holds for every finite sequence of scalars $\{c(\lambda)\}_{\lambda \in B}$.

Lemma 7. Given $\mathcal{S} \subset \mathbb{T}$ of positive measure, there exist a constant $\gamma=\gamma(\mathcal{S})>0$ for which the following holds: For infinitely many $n \in \mathbb{N} \gamma$ is a lower Riesz bound $\left(\right.$ in $\left.L^{2}(\mathcal{S})\right)$ for $E\left(B_{n}\right)$.

Proof. Let $\mathcal{S} \subset \mathbb{T}$, with $|\mathcal{S}|>0$. Applying Corollary 6 to the sequence $\{a(n)\}_{n \in \mathbb{N}}:=$ $\left\{\left|\widehat{\mathbb{1}_{\mathcal{S}}}(n)\right|^{2}\right\}_{n \in \mathbb{N}}\left(\right.$ where $\mathbb{1}_{\mathcal{S}}$ is the indicator function of the set $\mathcal{S}$ ), we get for every $\varepsilon>0$ infinitely many $n \in \mathbb{N}$ for which (3) holds. We write

$$
\begin{aligned}
\int_{\mathcal{S}}\left|\sum_{\lambda \in B_{n}} c(\lambda) e^{i \lambda t}\right|^{2} \frac{d t}{2 \pi}=\int_{\mathcal{S}}\left(\sum_{\lambda \in B_{n}}|c(\lambda)|^{2}+\sum_{\substack{\lambda, \mu \in B_{n} \\
\lambda \neq \mu}} c(\lambda) \overline{c(\mu)} e^{i(\lambda-\mu) t}\right) \frac{d t}{2 \pi}= \\
=|\mathcal{S}| \sum_{\lambda \in B_{n}}|c(\lambda)|^{2}+\sum_{\substack{\lambda, \mu \in B_{n} \\
\lambda \neq \mu}} c(\lambda) \overline{c(\mu) \widehat{\mathbb{1}_{\mathcal{S}}}}(\mu-\lambda) .
\end{aligned}
$$


By Cauchy-Schwarz inequality, the last term does not exceed

$$
\begin{gathered}
\left|\sum_{\substack{\lambda, \mu \in B_{n} \\
\lambda \neq \mu}} c(\lambda) \overline{c(\mu)} \widehat{\mathbb{1}_{\mathcal{S}}}(\mu-\lambda)\right| \leq\left(\sum_{\lambda, \mu \in B_{n}}|c(\lambda) \overline{c(\mu)}|^{2}\right)^{1 / 2}\left(\sum_{\substack{\lambda, \mu \in B_{n} \\
\lambda \neq \mu}}\left|\widehat{\mathbb{1}_{\mathcal{S}}}(\mu-\lambda)\right|^{2}\right)^{1 / 2}= \\
=\sum_{\lambda \in B_{n}}|c(\lambda)|^{2}\left(\sum_{\substack{\lambda, \mu \in B_{n} \\
\lambda \neq \mu}}\left|\widehat{\mathbb{1}_{\mathcal{S}}}(\mu-\lambda)\right|^{2}\right)^{1 / 2} .
\end{gathered}
$$

By (3) we get

$$
\sum_{\substack{\lambda, \mu \in B_{n} \\ \lambda \neq \mu}}\left|\widehat{\mathbb{1}_{\mathcal{S}}}(\mu-\lambda)\right|^{2}=2 \sum_{\substack{\lambda, \mu \in B_{n} \\ \mu<\lambda}}\left|\widehat{\mathbb{1}_{\mathcal{S}}}(\mu-\lambda)\right|^{2}<2 \varepsilon
$$

hence

$$
\int_{\mathcal{S}}\left|\sum_{\lambda \in B_{n}} c(\lambda) e^{i \lambda t}\right|^{2} \frac{d t}{2 \pi} \geq\left(|\mathcal{S}|-(2 \varepsilon)^{1 / 2}\right) \sum_{\lambda \in B_{n}}|c(\lambda)|^{2} \geq \frac{|\mathcal{S}|}{2} \sum_{\lambda \in B_{n}}|c(\lambda)|^{2} .
$$

Fixing some $\varepsilon<\frac{|\mathcal{S}|^{2}}{8}$, we get the last inequality holds for infinitely many $n \in \mathbb{N}$.

The next lemma shows how to combine blocks which correspond to different progressions.

Lemma 8. Let $\gamma>0, \mathcal{S} \subset \mathbb{T}$ with $|\mathcal{S}|>0$, and $A_{1}, A_{2} \subset \mathbb{N}$ finite subsets s.t $\gamma$ is a lower Riesz bound (in $\left.L^{2}(\mathcal{S})\right)$ for $E\left(A_{j}\right), j=1,2$. Then for any $0<\gamma^{\prime}<\gamma$ there exists $M \in \mathbb{Z}$ s.t. the system $E\left(A_{1} \cup\left(M+A_{2}\right)\right)$ has $\gamma^{\prime}$ as a lower Riesz bound.

Proof. Denote $P_{j}(t)=\sum_{\lambda \in A_{j}} c_{j}(\lambda) e^{i \lambda t}, j=1,2$. Notice that for sufficiently large $M=M(\mathcal{S})$, the polynomials $P_{1}$ and $e^{i M t} P_{2}$ are "almost orthogonal" on $\mathcal{S}$, meaning

$$
\int_{\mathcal{S}}\left|P_{1}(t)+e^{i M t} \cdot P_{2}(t)\right|^{2} \frac{d t}{2 \pi}=\left\|P_{1}\right\|_{L^{2}(\mathcal{S})}^{2}+\left\|P_{2}\right\|_{L^{2}(\mathcal{S})}^{2}+o(1)
$$


where the last term is uniform w.r. to all polynomials having $\|P\|_{L^{2}(\mathbb{T})}=1$.

Now we are ready to finish the proof of Theorem 2.

Given $\mathcal{S}$ take $\gamma$ from Lemma 7 and denote by $\mathcal{N}$ the set of all natural numbers $n$ for which $\gamma$ is a lower Riesz bound (in $L^{2}(\mathcal{S})$ ) for $E\left(B_{n}\right)$. Define

$$
\Lambda=\bigcup_{n \in \mathcal{N}}\left(M_{n}+B_{n}\right)
$$

Due to Lemma 8 we can define subsequently for every $n \in \mathcal{N}$, an integer $M_{n}$ s.t. for any partial union

$$
\Lambda(N)=\bigcup_{\substack{n \in \mathcal{N} \\ n<N}}\left(M_{n}+B_{n}\right), N \in \mathcal{N}
$$

the corresponding exponential system $E(\Lambda(N))$ has lower Riesz bound $\frac{\gamma}{2} \cdot\left(1+\frac{1}{N}\right)$, so we get that $E(\Lambda)$ is a $\operatorname{RS}$ in $L^{2}(\mathcal{S})$.

\section{Proof of theorem 3}

In order to obtain $\Lambda$ which satisfies property $\left(i^{\prime}\right)$ we will require the following result.

Theorem A. (1], Thm. 13.12) Let $d(n)$ denote the number of divisors of an integer $n$. Then $d(n)=o\left(n^{\varepsilon}\right)$ for every $\varepsilon>0$.

The next lemma will be used to control the contribution of blocks when they are not disjoint.

Lemma 9. Let $\{a(n)\}_{n \in \mathbb{N}}$ a sequence of non-negative numbers s.t. $\sum_{n=1}^{\infty} a(n) \leq 1$. Then for every $\alpha>1$ there exist $\varepsilon(\alpha)>0$ and $\nu(\alpha) \in \mathbb{N}$ s.t. for every $N \geq \nu(\alpha)$ 
one can find an integer $\ell_{\alpha, N}<N^{\alpha}$ satisfying

$$
\sum_{n=1}^{N} a\left(n \ell_{\alpha, N}\right)<\frac{1}{N^{1+\varepsilon(\alpha)}} .
$$

Proof. Fix $\alpha>1$ and apply Theorem A with $\varepsilon$ small enough, depending on $\alpha$, to be chosen later. We get the inequality $d(k)<k^{\varepsilon}$ holds for every $k \geq \nu(\alpha)$. Fix $N \geq \nu(\alpha)$, and notice that for every $L \in \mathbb{N}$

$$
\sum_{\ell=1}^{L} \sum_{n=1}^{N} a(n \ell) \leq \sum_{k=1}^{L N} d(k) a(k)<(L N)^{\varepsilon} .
$$

It follows that there exists an integer $0<\ell<L$ s.t.

$$
\sum_{n=1}^{N} a(n \ell)<\frac{(L N)^{\varepsilon}}{L}=\frac{N^{\varepsilon}}{L^{1-\varepsilon}} .
$$

In order to get (4) we ask

$$
\frac{N^{\varepsilon}}{L^{1-\varepsilon}}<\frac{1}{N^{1+\varepsilon}}
$$

which yields

$$
N^{\frac{1+2 \varepsilon}{1-\varepsilon}}<L
$$

Therefore, choosing $\varepsilon=\varepsilon(\alpha)$ sufficiently small we see that $L$ may be chosen to be smaller than $N^{\alpha}$.

Setting

$$
B_{\alpha, N}:=\left\{\ell_{\alpha, N}, 2 \ell_{\alpha, N}, \ldots, N \ell_{\alpha, N}\right\}
$$

we get

Corollary 10. Let $\{a(n)\}_{n \in \mathbb{N}}$ be as in Lemma 9. For every $\alpha>1$ and $N \geq \nu(\alpha)$

$$
\sum_{\substack{\lambda, \mu \in B_{\alpha, N} \\ \mu<\lambda}} a(\lambda-\mu)<\frac{1}{N^{\varepsilon(\alpha)}} .
$$


The proof is identical to that of Corollary 6 .

We combine our estimates.

Lemma 11. Given $\mathcal{S} \subset \mathbb{T}$ of positive measure, there exist a constant $\gamma=\gamma(\mathcal{S})>0$ s.t. for every $\alpha>1$ there exists $N(\alpha) \in \mathbb{N}$ for which the following holds: For every integer $N \geq N(\alpha)$ one can find $\ell_{\alpha, N} \in \mathbb{N}$ satisfying $\ell_{\alpha, N}<N^{\alpha}$ and $\gamma$ is a lower Riesz bound $\left(\right.$ in $\left.L^{2}(\mathcal{S})\right)$ for $E\left(B_{\alpha, N}\right)$.

Proof. Let $\mathcal{S} \subset \mathbb{T}$, with $|\mathcal{S}|>0$. We fix $\alpha>1$ and apply corollary 10 to the sequence $\{a(n)\}_{n \in \mathbb{N}}:=\left\{\left|\widehat{\mathbb{1}_{\mathcal{S}}}(n)\right|^{2}\right\}_{n \in \mathbb{N}}$, we get $\varepsilon(\alpha)$ and for every $N \geq \nu(\alpha)$ a positive integer $\ell_{\alpha, N}<N^{\alpha}$ satisfying (5). Proceeding as in the proof of Lemma 7 we get

$$
\int_{\mathcal{S}}\left|\sum_{\lambda \in B_{\alpha, N}} c(\lambda) e^{i \lambda t}\right|^{2} d t \geq\left(|\mathcal{S}|-\frac{C}{N^{\varepsilon(\alpha) / 2}}\right) \sum_{\lambda \in B_{\alpha, N}}|c(\lambda)|^{2} \geq \frac{|\mathcal{S}|}{2} \sum_{\lambda \in B_{\alpha, N}}|c(\lambda)|^{2},
$$

where last inequality holds for all $N \geq N(\alpha)$.

For the last step of the proof we will use a diagonal process.

Given $\mathcal{S}$ find $\gamma$ using Lemma 11. This provides, for every $\alpha>1$ and every $N \geq N(\alpha)$, a block $B_{\alpha, N}$ s.t. $\gamma$ is a lower Riesz bound (in $L^{2}(\mathcal{S})$ ) for $E\left(B_{\alpha, N}\right)$. Let $\alpha_{k} \rightarrow 1$ be a decreasing sequence. Define

$$
\Lambda=\bigcup_{k \in \mathbb{N}} \bigcup_{N=N\left(\alpha_{k}\right)}^{N\left(\alpha_{k+1}\right)-1}\left(M_{N}+B_{\alpha_{k}, N}\right)
$$

Again, by Lemma 8, we can make sure any partial union has lower Riesz bound not smaller than $\frac{\gamma}{2}$, and so $E(\Lambda)$ is a $\operatorname{RS}$ in $L^{2}(\mathcal{S})$.

It follows directly from the construction that for every $N \in \mathbb{N} \Lambda$ contains an arithmetic progression of length $N$ and step $\ell<C(\alpha) N^{\alpha}$, for any $\alpha>1$, as required. 


\section{REFERENCES}

[1] T.M. Apostol, Introduction to analytic number theory, Springer, Berlin, 1976.

[2] J. Bourgain and L. Tzafriri, Invertibility of "large" submatrices with applications to the geometry of Banach spaces and harmonic analysis, Israel J. Math. 57 (1987), no. 3, 137-224.

[3] M. Bownik and D. Speegle, The Feichtinger conjecture for wavelet frames, Gabor frames and frames of translates, Canad. J. Math. 58 (2006), no. 6, 1121-1143.

[4] P.G. Casazza, M. Fickus, J.C. Tremain and E. Weber, The Kadison-Singer problem in mathematics and engineering: a detailed account, In: Operator theory, operator algebras, and applications, volume 414 of Contemp. Math., 299-355. Amer. Math. Soc., Providence, RI, 2006.

[5] W. Lawton, Minimal sequences and the Kadison-Singer problem, Bulletin of the Malaysian Mathematical Sciences Society, http://math. usm. my/bulletin,(2) 33 (2010), no. 2, 169-176.

[6] A. Marcus, D.A. Spielman and N. Srivastava, Interlacing Families II: Mixed Characteristic Polynomials and the Kadison-Singer Problem, arXiv:1306.3969, (2013).

[7] I.M. Miheev, On lacunary series, Mathematics of the USSR-Sbornik 27 (1975), no. 4, 481-502.

[8] A. Olevskiu and A. Ulanovskii, Universal sampling and interpolation of bandlimited signals, Geom. Funct. Anal. 18 (2008), no. 3, 1029-1052.

[9] A. Zygmund, Trigonometric series. 2nd ed. Vol. I, Cambridge University Press, New York, 1959.

School of Mathematical Sciences, Tel-Aviv University, Tel-Aviv 69978, Israel.

E-mail address: itaylond@post.tau.ac.il

E-mail address: olevskii@post.tau.ac.il 